\title{
Anterior Pelvic Exenteration for Chemo-irradiated Non-diethylstilbestrol Exposed Clear Cell Vaginal Cancer
}

\author{
NICOLAE BACALBASA ${ }^{1,2,3}$, IRINA BALESCU $^{4}$, MIHAELA VILCU $^{1,2}$, SIMONA DIMA $^{3}$ and IULIAN BREZEAN ${ }^{1,2}$ \\ 1 "Carol Davila" University of Medicine and Pharmacy, Bucharest, Romania; \\ 2 “I. Cantacuzino” Clinical Hospital, Bucharest, Romania; \\ 3 “Fundeni” Clinical Institute - Center of Excellence in Translational Medicine, Bucharest, Romania; \\ 4 "Ponderas" Academic Hospital, Bucharest, Romania
}

\begin{abstract}
Background/Aim: Clear cell vaginal adenocarcinomas are rare tumors occurring in women which are usually treated by chemo radiotherapy with good outcomes. However, in certain cases, this treatment is not associated with complete response and a further surgery is needed. Case Report: We present the case of a 38-year-old patient diagnosed with stage IVA clear cell vaginal cancer who had been previously submitted to radio chemotherapy and in whom the lesion persisted after the oncological treatment; therefore, the patient was proposed for surgery with curative intent. The tumor was resected by performing an anterior pelvic exenteration with good outcomes, the patient being discharged in the seventh postoperative day. At one-year follow-up the patient remains free of recurrent disease. Conclusion: Pelvic exenteration with curative intent might be the option of choice for persistent locally advanced clear cell vaginal cancer.
\end{abstract}

Vaginal cancer is a rare tumor, accounting for less than $2 \%$ of all gynecological malignancies, the most commonly encountered subtype being represented by squamous cell carcinoma (1). When it comes to clear cell vaginal adenocarcinoma, it represents a usual subtype of vaginal cancer, which is most commonly encountered in patients with prior history of diethylstilbestrol exposure. Patients diagnosed with this histopathological subtype in which a history of diethylstilbestrol exposure is not documented are even less frequently encountered, rare cases being reported

This article is freely accessible online.

Correspondence to: Irina Balescu, "Ponderas" Academic Hospital, Bucharest, Romania. Tel: +40 724077709, e-mail: irina.balescu@ ponderas-ah.ro

Key Words: Clear cell vaginal cancer, anterior pelvic exenteration, neoadjuvant chemo-irradiation. so far (1-4). In consequence, data regarding the most efficient therapeutic protocol are scarce; however, it seems that a combination of external radiotherapy, intracavitary brachytherapy and concurrent platinum-based chemotherapy might provide a good local control of the disease. Moreover, there are cases in which a total remission of the tumor could be achieved by using this therapeutic strategy (5). However, cases presenting with per primam locally advanced tumors might need extended pelvic resections in order to achieve the local control of the pelvic malignancy.

\section{Case Report}

We present the case of a 38-year-old female diagnosed with locally advanced clear cell vaginal carcinoma who was submitted to chemo-radiation followed by anterior pelvic exenteration.

The 38-year-old patient presented herself in December 2017 to the gynecologist for the apparition of irregular vaginal bleeding in association with hematuria. At that moment the local examination revealed the presence of a vaginal tumor developed at the level of the anterior vaginal wall measuring approximately $6 \mathrm{~cm}-$ in length, $5 \mathrm{~cm}$ in width and $4 \mathrm{~cm}$ in depth, invading the whole length of the urethra; the rectal touch infirmed the presence of clinical signs of local invasion. The vaginal tumor was further on biopsied and proved to be a high-grade clear cell vaginal adenocarcinoma. The computed tomography performed at that moment excluded the presence of distant metastases and confirmed the presence of a tumoral mass of 58/47/58 $\mathrm{mm}$ developed at the level of the anterior vaginal wall invading the posterior wall of the urinary bladder (including the mucosal layer of the bladder), both levator ani muscles, and the urethra. This result was confirmed by pelvic magnetic resonance imaging, which described the presence of a $49 / 51 / 58 \mathrm{~mm}$ mass invading the posterior wall of the urinary bladder (at the level of the trigon) as well as the urethra. The patient was submitted to 
neoadjuvant radiotherapy consisting of 20 fractions of 2 Grays each (a total dose of 40 Grays) followed by six cycles of paclitaxel and cisplatin. At the end of the neoadjuvant treatment the magnetic resonance imaging which was performed in June 2018 demonstrated the persistence of the tumor which measured at this moment $33 / 34 / 55 \mathrm{~mm}$. In addition, the invasion at the level of the urinary bladder trigon persisted, so the patient was submitted to surgery after obtaining a written consent in July 2018. Intraoperatively, the presence of urinary bladder involvement was confirmed, so the patient was submitted to anterior pelvic exenteration. Due to the previous history of external radiotherapy, no urinary tract reconstruction was performed, the two ureters being exteriorized in right side terminal cutaneous ureterostomy. Intraoperative aspects are shown in Figures 1-4. The postoperative outcome was uneventful, the patient being discharged in the seventh postoperative day. The histopathological studies confirmed the presence of a poorly differentiated clear cell vaginal adenocarcinoma as well as the invasion at the level of the urethra and of the urinary trigon. In the meantime, negative resection margins were also confirmed. At one-year follow-up the patient is free of any local or distant relapse.

\section{Discussion}

Initially considered as an incurable gynecologic malignancy, vaginal carcinoma accounts for up to $2 \%$ of all vaginal cancers and are mainly represented by squamous cell carcinomas. Vaginal clear cell carcinomas account for less than $10 \%$ of all vaginal carcinomas and are most commonly related to diethylstilbestrol in utero use (1-2). This correlation has been widely demonstrated, most studies demonstrating a high incidence of this malignancy among daughters of women who used this synthetic molecule of estrogen during the first 18 weeks of gestation. The explanation is related to the interference between this synthetic product and the Mullerian epithelium degeneration at the level of the fetal vagina; therefore, most cases diagnosed with this malignancy are usually young females (during the first decades of life) (oppositely to squamous cell carcinoma which is usually diagnosed in patients between the fifth and seventh decades of age) (5-8). However, in the absence of a previous history of diethylstilbestrol use, clear cell vaginal cancer had been rarely encountered, most often being related with patients presenting congenital malformations of the uterus or vagina such as bicornuate uterus $(3,9)$. When it comes to the location of clear cell vaginal adenocarcinomas, they are usually encountered at the level of the upper third of the anterior wall of the vagina; however, in less than $10 \%$ of cases, the entire length of the vaginal wall is involved.
Therefore, the particularity of our case was related to the absence of a previous history of in utero diethylstilbestrol use, to the absence of any other congenital malformations of the gynecological tract as well as to the relatively advanced age at diagnosis (38 years in our case while the median reported age is 18 years) (3).

As for the therapeutic strategy, we also decided to start with radio chemotherapy with curative intent. However, the tumoral lesion reported only a slight regression in the volume; therefore, surgery with curative intent was needed in order to provide a chance to control the disease.

One of the first studies which were conducted on this issue was published in 1979 and included 346 patients with clear cell adenocarcinomas of the uterine cervix and of the vagina, a history of diethylstilbestrol use being demonstrated in two thirds of these cases (10); among the 346 patients, there were 189 cases diagnosed with vaginal tumors. As expected, the long-term outcomes of these patients proved to be significantly influenced by the stage at diagnosis; while for stage I patients the five year survival rate was $87 \%$ for cases with vaginal cancer and $91 \%$ for those with cervical cancer, for stage IV patients the overall survival significantly decreased, a single case of the five patients diagnosed in this stage of disease reporting a long-term survival of 27 months (10).

However, since extended pelvic resections have been widely performed in order to treat locally advanced or relapsed cervical tumors, this type of surgery was also successfully implemented in order to treat persistent vaginal cancer. In such cases the usual development of the tumor on the anterior wall of the vagina might impose performing an anterior pelvic exenteration. Experience gained during treating stage IV cervical cancer with urinary bladder invasion or pelvic recurrences after surgically treated cervical cancer provided the opportunity to offer a radical treatment in such cases (11-14).

An interesting study which came to demonstrate the effectiveness of pelvic exenteration for clear cell adenocarcinoma of the uterine cervix and of the vagina was published in 1989 by Senekjian et al. The study included 29 cases, eight of these patients being submitted to surgery for lesions which persisted after radiotherapy (15).

As it can be observed, most studies conducted on this issue have been published between 1970 and $1990(15,16)$. This fact can be also correlated with the relationship between clear cell vaginal carcinoma and diethylstilbestrol use. The first year in which this product was widely introduced in order to prevent miscarriages and other pregnancy related problems was 1947 for the United States of America. The product was widely used in the United States until 1971, when the Food and Drug Administration organization declared the use of this product as contraindicated during pregnancy. Therefore, a significant decrease in the number of clear cell vaginal carcinoma was encountered in women who were born after this year (17). 


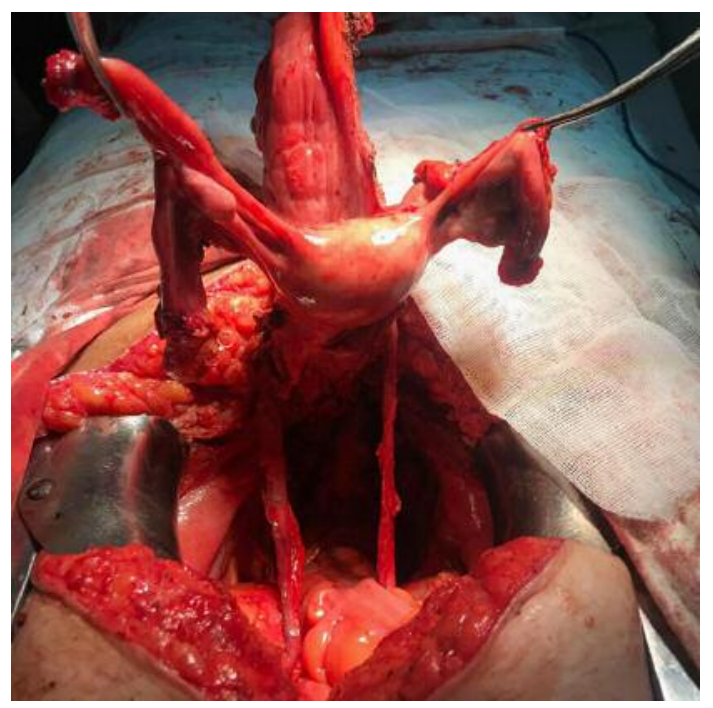

Figure 1. The aspect after mobilization of the tumor en bloc with total hysterectomy, bilateral adnexectomy and total cystectomy.

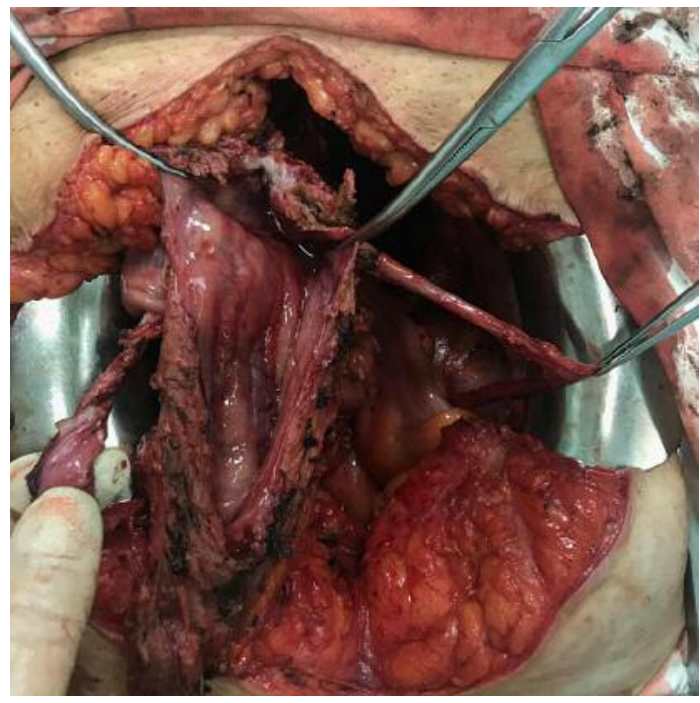

Figure 2. Intraoperative aspect demonstrating the presence of tumoral invasion at the level of the urinary bladder trigon.

\section{Conclusion}

In conclusion, non-related to diethylstilbestrol in utero use vaginal clear cell carcinomas are rare eventualities, only rare cases being reported so far. The particularities of our case are related to the relatively advanced age at the time of diagnosis, to the absence of exposure to diethylstilbestrol use as well as to the absence of association of any congenital gynecologic abnormalities. As for the topical particularities,

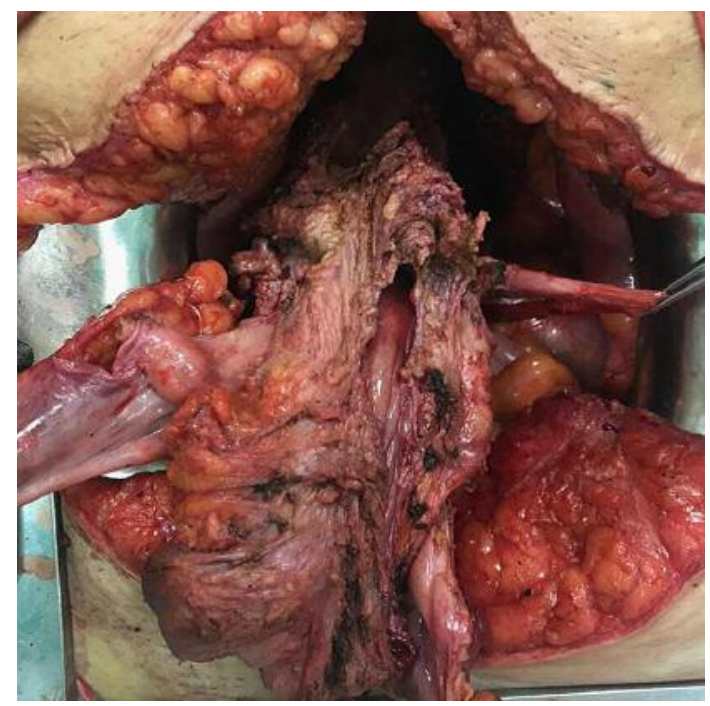

Figure 3. The aspect after mobilization of the urinary bladder.

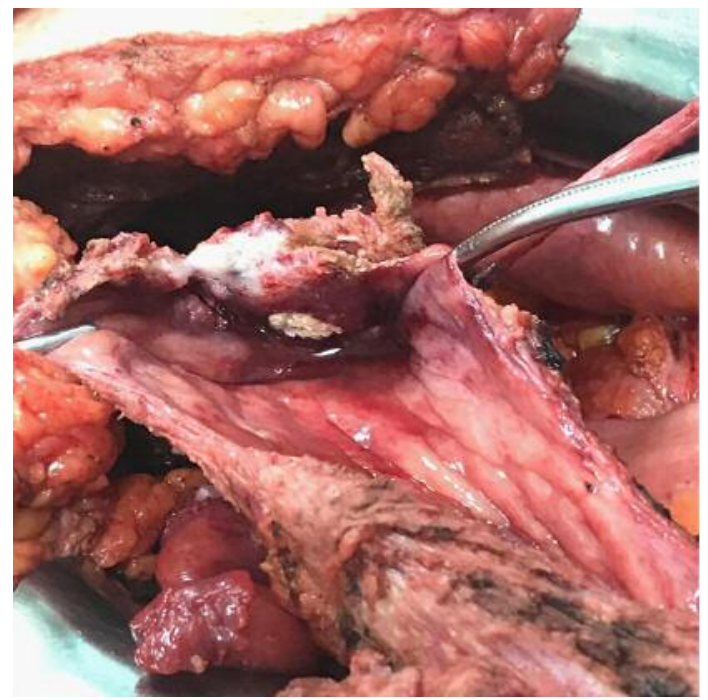

Figure 4. The vaginal tumor invading the posterior wall of the urinary bladder.

they are represented by the dimension of the tumor, which initially invaded the whole length of the anterior vaginal wall as well as the entire length of the posterior urethra and the urinary bladder trigon. Although chemo-irradiation was performed, the tumor persisted, therefore, radial pelvic surgery consisting of an anterior pelvic exenteration was taken into consideration and proved to be a valid option. At one-year follow-up, the patient is free of any recurrent local or distant lesion. 


\section{Conflicts of Interest}

The Authors have no conflicts of interest to declare regarding this study.

\section{Authors' Contributions}

NB - Performed surgical procedure; IB - prepared the manuscript; $\mathrm{SD}$ - produced figures; MV, IB - part of the surgical team; IB adviced about surgical oncology procedure, revised the final draft of the manuscript.

\section{Acknowledgements}

This work was supported by the project entitled „Multidisciplinary Consortium for Supporting the Research Skills in Diagnosing, Treating and Identifying Predictive Factors of Malignant Gynecologic Disorders", project number PN-III-P1-1.2-PCCDI2017-0833

\section{References}

1 Creasman WT, Phillips JL and Menck HR: The National Cancer Data Base report on cancer of the vagina. Cancer 83: 1033-1040, 1998. PMID: 9731908.

2 Hanselaar A, van Loosbroek M, Schuurbiers O, Helmerhorst T, Bulten $\mathrm{J}$ and Bernhelm $\mathrm{J}$ : Clear cell adenocarcinoma of the vagina and cervix. An update of the central Netherlands registry showing twin age incidence peaks. Cancer 79: 2229-2236, 1997. PMID: 9179071. DOI: 10.1002/(sici)1097-0142(19970601) 79:11<2229::aid-cncr22>3.0.co;2-x

3 Tanaka H, Tabata T, Yanase H, Nishiyama M, Yamawaki T, Taniguchi $\mathrm{H}$ and Toyoda $\mathrm{N}$ : Clear cell adenocarcinoma of the vagina in a young female, treated by combination chemotherapy (local and systemic chemotherapy), complicated with chromosomal abnormality. Gynecol Oncol 55: 259-264, 1994. PMID: 7959294. DOI: 10.1006/gyno.1994.1287

4 Uehara T, Onda T, Sasajima Y, Sawada M and Kasamatsu T: A case of vaginal clear cell adenocarcinoma complicated with congenital anomalies of the genitourinary tract and metanephric remnant without prenatal diethylstilbestrol exposure. J Obstet Gynaecol Res 36: 681-685, 2010. PMID: 20598057. DOI: 10.1111/j.1447-0756.2010.01178.x

5 Ikeda Y, Oda K, Aburatani H, Kawana K, Osuga Y and Fujii T: Non-diethylstilbestrol exposed vaginal clear cell adenocarcinoma has a common molecular profile with ovarian clear cell adenocarcinoma: A case report. Gynecol Oncol Rep 10: 49-52, 2014. PMID: 26082939. DOI: 10.1016/j.gynor.2014.05.006

6 Nordqvist SR, Fidler WJ Jr., Woodruff JM and Lewis JL Jr.: Clear cell adenocarcinoma of the cervix and vagina. A clinicopathologic study of 21 cases with and without a history of maternal ingestion of estrogens. Cancer 37: 858-871, 1976. PMID: 1253110. DOI: 10.1002/1097-0142(197602)37:2<858: :aid-cncr2820370235>3.0.co;2-8
7 Herbst AL and Scully RE: Adenocarcinoma of the vagina in adolescence. A report of 7 cases including 6 clear-cell carcinomas (so-called mesonephromas). Cancer 25: 745-757, 1970. PMID: 5443099. DOI: 10.1002/1097-0142(197004) 25:4<745::aid-cncr2820250402>3.0.co;2-2

8 Herbst AL, Norusis MJ, Rosenow PJ, Welch WR and Scully RE: An analysis of 346 cases of clear cell adenocarcinoma of the vagina and cervix with emphasis on recurrence and survival. Gynecol Oncol 7: 111-122, 1979. PMID: 437563. DOI: 10.1016/0090-8258(79)90087-8

9 Guzin K, Eser SK, Yigit A and Zemher Z: Primary clear cell carcinoma of the vagina that is not related to in utero diethylstilbestrol use. Gynecol Surg 3: 281-284, 2006. DOI: 10.1007/s10397-006-0182-4

10 Satou Y and Takasu K: Clear cell adenocarcinoma in duplicated and imperforated vagina with didelphys uterus - A case report. J Kyoto Pref Univ Med 99: 725-738, 1990.

11 Bacalbasa $\mathrm{N}$ and Balescu I: Palliative pelvic exenteration for pelvic recurrence invading the sciatic foramen with chronic cutaneous perineal fistula after radical surgery for cervical cancer: a case report. Anticancer Res 35: 4877-4880, 2015. PMID: 26254382.

12 Bacalbasa N and Balescu I: Partial cystectomy for atypical isolated recurrence of ovarian adenocarcinoma - a case report and literature review. In Vivo 31: 429-433, 2017. PMID: 28438874. DOI: 10.21873 /invivo. 11078

13 Bacalbasa N, Balescu I and Tomescu D: Partial cystectomy with distal ureteral resection and re-implantation for locally invasive cervical cancer. Anticancer Res 35: 5539-5542, 2015. PMID: 26408722.

14 Bacalbasa N, Tomescu D and Balescu I: Use of inflated foley catheters to prevent early empty pelvis complications following pelvic exenteration. Anticancer Res 35: 5543-5546, 2015. PMID: 26408723.

15 Senekjian EK, Frey K and Herbst AL: Pelvic exenteration in clear cell adenocarcinoma of the vagina and cervix. Gynecol Oncol 34: 413-416, 1989. PMID: 2767532. DOI: 10.1016/00908258(89)90183-2

16 Abitbol A, Straus MJ, Sedlis A and Davidian M: Multimodality treatment of adenocarcinoma of the vagina Stage IV: potential for cure. Gynecol Oncol 16: 296-298, 1983. PMID: 6313495. DOI: 10.1016/0090-8258(83)90106-3

17 Huo D, Anderson D, Palmer JR and Herbst AL: Incidence rates and risks of diethylstilbestrol-related clear-cell adenocarcinoma of the vagina and cervix: Update after 40-year follow-up. Gynecol Oncol 146: 566-571, 2017. PMID: 28689666. DOI: 10.1016/j.ygyno.2017.06.028
Received September 1, 2019

Revised September 14, 2019

Accepted September 16, 2019 